\title{
WASHINGTON, D.C.
}

$$
\text { June 19, } 1925 .
$$

\section{ADDRESSES CF SOURCES OF PUBI ICATIONS}

\section{ISSUED BY THE LIME. GYPSUM AND SAND-IIME ERIUR SECTION}

National Lime Association,

9io G St., iN.w.,

Washington, D.C.

The Gypsum Industries,

844 Rush street,

Chicago, III.

Sand-Lime Brick Asbociation,

(Attn: Mir.J. Morley Zancer, Fresident),

Sa̧inaw, Mich.

American Ceramic Society,

Lorci Hall,

Ohio State University,

Columbus, Onio.

United Statcs Geological Survey,

Departinent of the Interior,

Washington, D.C.

American Societ; for Testing Naterials,

1315 Spruce Street, Philadelphia, Pa.

Engineering News,

EnEireering Record,

Engineering News-Record,

Chemical and Metallurgical Engineering,

NcGrar-Hill Co.,

loth Ave. at 36 th st.,

New York, N.Y.

Journal of Industrial and Engineering Chemistry,

810-18th St., N.莉.,

Washington, D.C.

United States Patent office,

Department of the Interior,

Washington, D.C. 

Letter Circular 168 - June 19, 1925.

Rock Prodicts,

Tiadspress Publishing Corp.,

542 s. Dearkom st.,

Chicago, Ill.

Scientiflc American Publishinr Co.,

Munn and Co.,

233 Broadmay,

New York, N.Y.

Concrete,

Concrete-Cement Age Publishing Co., Detroit, Mich.

Engineering and Contracting,

9 S. CIEnton St.,

Chicago, Ill.

Mining and scientific Press,

Dewey Publishing Co.,

420 Market street,

San Francisco, Calif.

Building Officials Conference,

Jeremiah Downey, Secretary,

City Hall,

Cambridge, Mass. 
